\title{
INDEX CLASSES FOR VECTOR FIELDS AND THEIR RELATION TO CERTAIN CHARACTERISTIC CLASSES
}

\author{
OSKAR HAMLET
}

\begin{abstract}
While studying vector fields on manifolds with boundary there are three important indexes to consider. We construct three cohomology classes to compute these. We relate these classes to other classes, the relative Euler class as defined by Sharafutdinov and the secondary Chern-Euler class as defined by Sha. Our results also yield a new proof of the Poincaré-Hopf index theorem.
\end{abstract}

\section{Introduction and results}

Let $M$ be a smooth, compact, oriented $m$-dimensional manifold with boundary $\partial M$ and $v$ a smooth vector field on $M$, nonvanishing on the boundary, with isolated zeros. Further let $I^{\circ}(v)$ denote the index of the vector field, $I^{-}(v)$ and $I^{+}(v)$ the index of the vector field restricted to and projected onto the boundary counting only those vectors for which $v$ pointed inwards respectively outwards. The Poincaré-Hopf theorem in its more general form states that $I^{\circ}(v)+I^{-}(v)=\chi(M)$, the Euler characteristic of $M$. The generalisation of the Poincaré-Hopf theorem to the case of manifolds with boundary is due to Morse [2] and was rediscovered and further studied by Pugh and Gottlieb [3], [1]. In this paper we will define three singular cohomology classes $u^{\circ}, u^{-}, u^{+}$ in $H^{m}\left(T M, T M_{0}^{\partial}\right)$ which are intimately related to this theorem. Here the superscript $\partial$ denotes restriction of the bundle to the boundary and the subscript 0 denotes the non-zero elements, the (co)homology considered throughout the paper is with respect to integer coefficients unless otherwise mentioned. We prove that these classes satisfy the following index theorem.

THEOREM 1.1. Let $\mu$ be the fundamental class of $M$ and $v$ a vector field, nonvanishing on the boundary, with isolated zeros. Then

$$
\begin{aligned}
I^{\circ}(v) & =\left\langle v^{*} u^{\circ}, \mu\right\rangle \\
I^{-}(v) & =\left\langle v^{*} u^{-}, \mu\right\rangle \\
I^{+}(v) & =\left\langle v^{*} u^{+}, \mu\right\rangle .
\end{aligned}
$$

Received 17 February 2011, in final form 18 February 2011. 
In view of this result it is quite natural to look for relations to other index classes. In [4] Sha defined a secondary Chern-Euler class $\Upsilon$ which can be viewed as an element of $H^{m-1}\left(T M_{0}^{\partial} ; \mathrm{Z}\left[\frac{1}{2}\right]\right)$. From the construction it will be clear that the classes $u^{-}$and $u^{+}$have natural preimages $\tilde{u}^{-}$and $\tilde{u}^{+}$in $H^{m-1}\left(T M_{0}^{\partial}\right)$. These preimages are the building blocks of the secondary ChernEuler class.

TheOREM 1.2. Sha's secondary Chern-Euler class is related to our index classes as follows: $2 \Upsilon=\tilde{u}^{+}-\tilde{u}^{-}$.

We also relate $u^{\circ}$ and $u^{-}$to the relative Euler class $e(E, n)$ as defined in [5] by Sharafutdinov. The latter is defined from the following data. An oriented $m$-plane vector bundle $\pi: E \rightarrow B$, a closed subset $A$ of $B$, and a nonvanishing section $n$ over $A$. Sharafutdinov proved that this class satisfies the following theorem.

THEOREM 1.3 (Sharafutdinov). Let $n: \partial M \rightarrow T M$ be an outwards-pointing vector field over the boundary of an oriented manifold $M$ with fundamental class $\mu$, then $\langle e(T M, n), \mu\rangle=\chi(M)$.

In this more general setting the definition of $u^{-}$will depend on the section $n$ and we use a subscript $n$ as reminder of that. We relate our classes to the relative Euler class as follows.

THeOrem 1.4. Let $v$ be a section of $E$ which is nonvanishing on A. Then $v^{*}\left(u^{\circ}+u_{n}^{-}\right)=e(E, n)$.

The Poincaré-Hopf index theorem is now an immediate corollary of Theorem 1.1 together with Theorem 1.3 and 1.4.

\section{Definitions}

Let $\pi: E \rightarrow B$ be a smooth oriented $m$-dimensional vector bundle over a topological space $B$. Let $A$ be a closed subset of $B$ and let $E^{A}$ be the restriction of $E$ to $A$. Assume that there exists a nonvanishing section $n: A \rightarrow E^{A}$. Denote by $\langle n\rangle$ the ray generated by $n, s:=-n$ and $\langle s\rangle$ the opposite ray. Using the commutative diagram below we will define the index classes. All maps in the diagram which we have not defined are to be understood as restriction or connecting homomorphisms for the relevant pair/triple of spaces. We will throughout the paper use diagrams in this way to specify the homomorphisms we need.

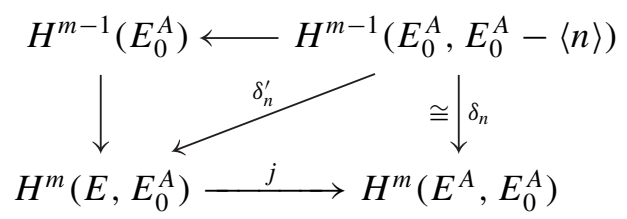


That $\delta_{n}$ is an isomorphism follows from $\left(E^{A}, E_{0}^{A}-\langle n\rangle\right)$ being homotopy equivalent as a pair to $(A, A)$. Replacing all $n$ 's in the diagram by $s$ 's allows us to define $\delta_{s}$ and $\delta_{s}^{\prime}$ in the same way. Further let $k$ be the restriction homomorphism $H^{m}\left(E, E_{0}\right) \rightarrow H^{m}\left(E, E_{0}^{A}\right)$. Let $u$ be the Thom class of $E$, keeping the notation we define

$$
\begin{aligned}
u^{\circ} & :=k u \\
u_{n}^{-} & :=-\delta_{s}^{\prime}\left(\delta_{s}\right)^{-1} j u^{\circ} \\
u_{n}^{+} & :=\delta_{n}^{\prime}\left(\delta_{n}\right)^{-1} j u^{\circ} .
\end{aligned}
$$

In the case where $(E, B, A)=(T M, M, \partial M)$ we will drop the subscript $n$, always choosing $n$ as an outwards-pointing vector field on the boundary. A rather simple argument which we omit shows that the index classes then does not depend on the choice of such $n$. As the upper triangle in the diagram commutes, $\delta_{n}^{\prime}$ and $\delta_{s}^{\prime}$ factors over $H^{m-1}\left(E_{0}^{A}\right)$ giving us the preimages $\tilde{u}^{-}$and $\tilde{u}^{+}$mentioned in the introduction.

\section{The relative Euler class}

With notation from the diagram below the relative Euler class is defined as $e(E, n):=\left(\pi^{*}\right)^{-1} t u$.

Proof OF Theorem 1.4. From their definitions it follows immediately that $u^{\circ}+u_{n}^{-} \in \operatorname{Ker} j$. Thus it has a unique preimage $\chi \in H^{m}\left(E, E^{A}\right)$. The following diagram describes the situation and specifies the homomorphisms we need for the proof. We have

$$
\begin{aligned}
& v^{*}\left(u^{\circ}+u_{n}^{-}\right)=v^{*} i \chi=\left(\pi^{*}\right)^{-1} \chi=\left(\pi^{*}\right)^{-1} h i \chi \\
& =\left(\pi^{*}\right)^{-1} h\left(u^{\circ}+u_{n}^{-}\right)=\left(\pi^{*}\right)^{-1} h u^{\circ}+\left(\pi^{*}\right)^{-1} r l u_{n}^{-} \\
& =\left(\pi^{*}\right)^{-1} h k u=\left(\pi^{*}\right)^{-1} t u=e(E, n) \text {. } \\
& H^{m-1}\left(E, E_{0}^{A}-\langle s\rangle\right) \\
& \cong \delta_{s}^{\prime} \\
& H^{m}\left(E, E^{A}\right) \longleftarrow H^{m}\left(E, E_{0}^{A}\right) \stackrel{j}{\longrightarrow} H^{m}\left(E^{A}, E_{0}^{A}\right)
\end{aligned}
$$

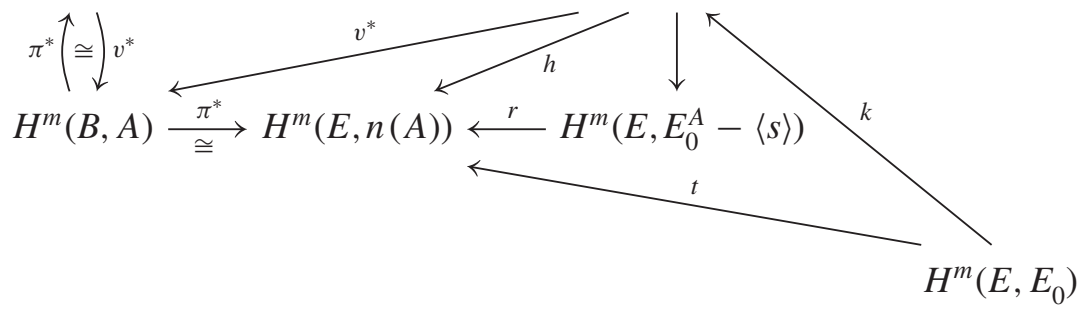




\section{The indexes}

We will divide the proof into two parts. In the first part we will prove the first statement that $I^{\circ}(v)=\left\langle v^{*} u^{\circ}, \mu\right\rangle$, a presumably known result which we have not been able to find in the literature. We will then use a modified version of this argument in the second part.

Proof of Theorem 1.1. Recall that for a vector field $v: \mathrm{R}^{m} \rightarrow T \mathrm{R}^{m}$ vanishing only at the origin we define the local index at 0 to be the degree of the $p_{2} \circ v: \mathrm{R}_{0}^{m} \rightarrow \mathrm{R}_{0}^{m}$ where $p_{2}: T \mathrm{R}_{0}^{m}=\mathrm{R}^{m} \oplus \mathrm{R}_{0}^{m} \rightarrow \mathrm{R}_{0}^{m}$ is the projection onto the second summand. We define the mapping degree of a map $f: \mathrm{R}_{0}^{m} \rightarrow \mathrm{R}_{0}^{m}$ by it's action on a generator $v$ of $H_{m-1}\left(\mathrm{R}_{0}^{m}\right)$, i.e., $f_{*} v=\operatorname{degree}(f) v$. In the general setting we have a vector field $v$ on an oriented manifold $M$ with isolated zeros $Z:=\{z \in M, v(z)=0\}$. For $z \in Z$ we choose local coordinates $\Phi$ with $\Phi(0)=z$ for a neighbourhood $U$ of $z$ such that $z$ is the only zero of $v$ in $U$. The local index is then defined as the degree of $p_{2} \circ d \Phi^{-1} \circ v \circ \Phi$. Since $\left(p_{2}\right)_{*}, \Phi_{*}$ and $d \Phi_{*}$ are isomorphisms we can, being careful about our choice of generators, consider just $v_{*}$. Using the long exact sequences for the homology of $(U, U-z)$ and $\left(T U, T U_{0}\right)$ we can instead consider $v_{*}: H_{m}(U, U-z) \rightarrow H_{m}\left(T U, T U_{0}\right)$. In the degree calculation using local coordinates we used the same generator for two copies of $H_{m-1}\left(\mathrm{R}_{0}^{m}\right)$. This corresponds to using generators of $H_{m}(U, U-z)$ and $H_{m}\left(T U, T U_{0}\right)$ corresponding to the same orientation of the manifold. We choose the first generator to be the restriction of the fundamental class $\mu_{z}$.The second is then the homology class $u_{*}^{z}$ corresponding to the restriction $u^{z}$ of the Thom class $u$ to $U$ via $\left\langle u^{z}, u_{*}^{z}\right\rangle=1$. We then get the local index by $\left\langle u^{z}, v_{*} \mu_{z}\right\rangle=\left\langle u^{z}, \operatorname{index}(v, z) u_{*}^{z}\right\rangle=\operatorname{index}(v, z)$.

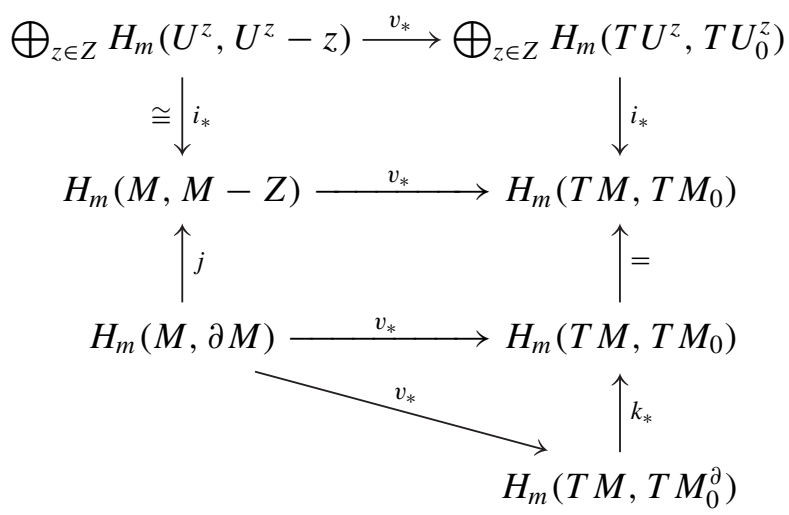

The global index $I^{\circ}(v)$ is defined to be the sum of the local indexes. Using the diagram above we calculate

$$
I^{\circ}(v)=\sum_{z \in Z}\left\langle u^{z}, v_{*} \mu_{z}\right\rangle=\sum_{z \in Z}\left\langle i^{*} u, v_{*} \mu_{z}\right\rangle=\sum_{z \in Z}\left\langle u, i_{*} v_{*} \mu_{z}\right\rangle
$$




$$
\begin{aligned}
& =\left\langle u, v_{*} i_{*} \sum_{z \in Z} \mu_{z}\right\rangle=\left\langle u, v_{*} j \mu\right\rangle=\left\langle u, v_{*} \mu\right\rangle \\
& =\left\langle u, k_{*} v_{*} \mu\right\rangle=\left\langle v^{*} k^{*} u, \mu\right\rangle=\left\langle v^{*} u^{\circ}, \mu\right\rangle .
\end{aligned}
$$

The boundary index $I^{-}(v)$ will be somewhat trickier since we only want to count "half" of the local indexes. We begin by establishing some notation.

Let $n$ be the outwards-pointing vector field used to define $u^{-}$. Each vector $w \in T M^{\partial}$ decomposes as $w=w_{\downarrow}+\lambda(w) n(\pi(w))$ with $w_{\downarrow} \in T \partial M$ and $\lambda \in$ R. Further let $Z^{-}:=\left\{z \in \partial M, v_{\downarrow}(z)=0, \lambda(v(z))<0\right\}$, i.e., the zeros of the projected vector field $v_{\downarrow}$ for which $v$ pointed inwards. Let $u_{\partial M}$ be the Thom class of $T \partial M$ and $\mu_{\partial M}$ the fundamental class of the boundary, both corresponding to the induced orientation of the boundary. Define $(+s):\left(T \partial M, T \partial M_{0}\right) \rightarrow\left(T M_{0}^{\partial}, T M_{0}^{\partial}-\langle s\rangle\right), v \mapsto v+s(\pi(v))$. By a simple homotopy argument $(+s)_{*}$ is seen to be an isomorphism between the corresponding homology groups.

Locally we can use the same method as in the interior case to calculate the indexes. Using the notation of the diagram below, for a $z \in Z^{-}$, index $\left(v_{\downarrow}, z\right)=$ $\left\langle u_{\partial M}, i_{*}\left(v_{\downarrow}\right)_{*}\left(\mu_{\partial M}\right)_{z}\right\rangle$. However we cannot use the same method to calculate the global index as before as this would also count the local indexes of zeros for which the original vector field points outwards. The trick lies in the following local factorisation of $\left(v_{\downarrow}\right)_{*}$ near inwards-zeros.

Lemma 4.1. Let $z \in Z^{-}$and $V$ be a neighbourhood of $z$ in $\partial M$ in which $z$ is the only zero of $v_{\downarrow}$. Then the restricted maps $\left(v_{\downarrow}\right)_{*}=(+s)_{*}^{-1} v_{*}: H_{m-1}(V, V-$ $z) \rightarrow H_{m-1}\left(T V, T V_{0}\right)$.

Proof. We can without loss of generality assume that $v$ points inwards in all of $V$. We can thus write $v=v_{\downarrow}+\lambda(v) n$ with $\lambda<0$ but this is obviously homotopy equivalent to $v_{\downarrow}-n=v_{\downarrow}+s$, hence $v$ is homotopy equivalent to $(+s) \circ v_{\downarrow}$.

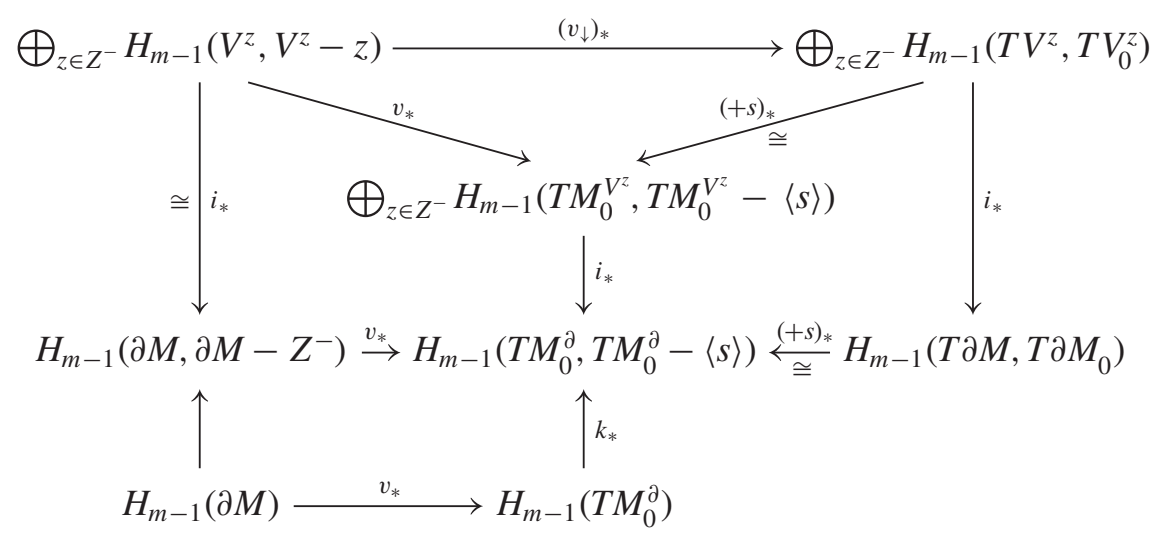


From the lemma it follows that the top triangle of the diagram above commutes. The rest of the diagram is trivially seen to commute. We calculate

$$
\begin{aligned}
I^{-}(v) & =\sum_{z \in Z^{-}}\left\langle u_{\partial M}, i_{*}\left(v_{\downarrow}\right)_{*}\left(u_{\partial M}\right)_{z}\right\rangle \\
& =\sum_{z \in Z^{-}}\left\langle u_{\partial M}, i_{*}(+s)_{*}^{-1} v_{*}\left(u_{\partial M}\right)_{z}\right\rangle \\
& =\left\langle u_{\partial M}, i_{*}(+s)_{*}^{-1} v_{*} \sum_{z \in Z^{-}}\left(u_{\partial M}\right)_{z}\right\rangle \\
& =\left\langle u_{\partial M},(+s)_{*}^{-1} v_{*} i_{*} \sum_{z \in Z^{-}}\left(u_{\partial M}\right)_{z}\right\rangle \\
& =\left\langle u_{\partial M},\left((+s)_{*}^{-1} v_{*} j u_{\partial M}\right)\right\rangle \\
& =\left\langle u_{\partial M},(+s)_{*}^{-1} k_{*} v_{*} \mu_{\partial M}\right\rangle \\
& =\left\langle v^{*} k^{*}\left((+s)^{*}\right)^{-1} u_{\partial M}, \mu_{\partial M}\right\rangle .
\end{aligned}
$$

Here we already have a very nice formulation of the boundary index and $k^{*}\left((+s)^{*}\right)^{-1} u_{\partial M}$ is an interesting index class. The remaining part consists of "moving" this index class to where it can meet the interior index class $u^{\circ}$. Define $\rho:\left(T M_{0}^{\partial}, T M_{0}^{\partial}-\langle s\rangle\right) \rightarrow\left(T M_{0}^{\partial}, T M_{0}^{\partial}-\langle n\rangle\right)$, as $\left(v_{\downarrow}+\lambda s\right) \mapsto\left(v_{\downarrow}-\lambda s\right)=$ $\left(v_{\downarrow}+\lambda n\right)$ i.e., a "fibrewise reflection" in the tangent plane of the boundary. The following diagram specifies the homomorphisms we will need for the rest of the proof.

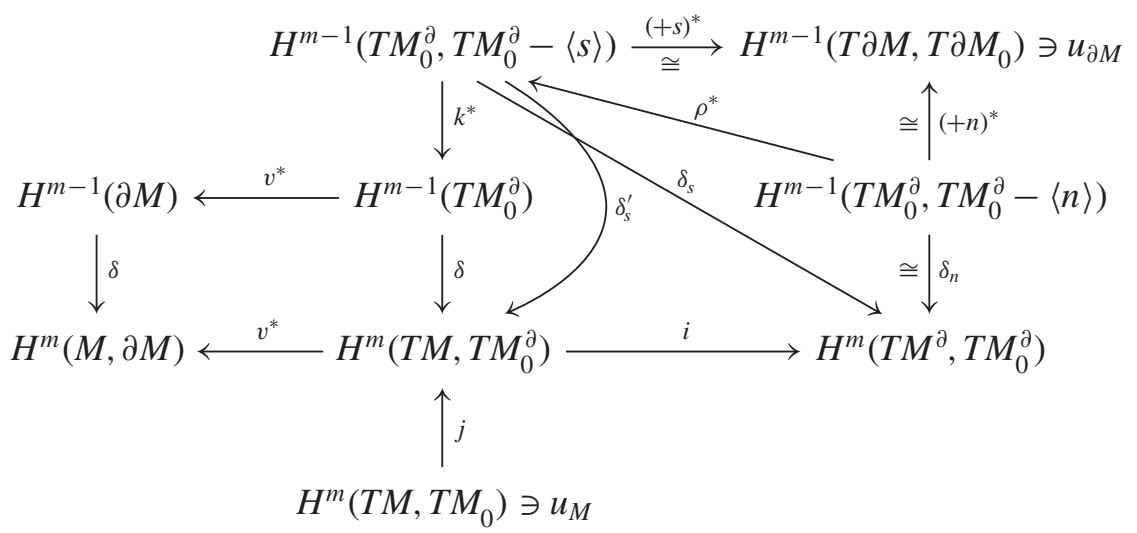

Using the diagram above we calculate

$$
\begin{aligned}
I^{-}(v) & =\left\langle v^{*} k^{*}\left((+s)^{*}\right)^{-1} u_{\partial M}, \mu_{\partial M}\right\rangle \\
& =\left\langle v^{*} k\left((+s)^{*}\right)^{-1}(+n)^{*} \delta_{n}^{-1} i j u_{M}, \mu_{\partial M}\right\rangle
\end{aligned}
$$




$$
\begin{aligned}
& =\left\langle-v^{*} k \delta_{s}^{-1} i j u_{M}, \mu_{\partial M}\right\rangle \\
& =\left\langle-\delta v^{*} k \delta_{s}^{-1} i j u_{M}, \mu_{M}\right\rangle=\left\langle-v^{*} \delta k \delta_{s}^{-1} i j u_{M}, \mu_{M}\right\rangle \\
& =\left\langle-v^{*} \delta_{s}^{\prime} \delta_{s}^{-1} i j u_{M}, \mu_{M}\right\rangle=\left\langle v^{*} u^{-}, \mu_{M}\right\rangle .
\end{aligned}
$$

The first equality just states what we already know, and the second follows from $u_{\partial M}=(+n)^{*} \delta_{n}^{-1} i j u_{M}$. The upper triangle commutes so $\left((+s)^{*}\right)^{-1}(+n)^{*}=$ $\rho^{*}$, we also have that $\rho^{*} \delta_{n}^{-1}=-\delta_{s}^{-1}$, from these facts the third equality follows. The fourth equality is a consequence of $\mu_{\partial M}=\partial \mu_{M}$ and $\delta=\partial^{*}$. The fifth uses that the leftmost rectangle commutes to switch the order of $\delta$ and $v^{*}$ and the sixth uses that $\delta k^{*}$ is a factorisation of $\delta_{s}^{\prime}$. Finally, the seventh equality follows from the definition of $u^{-}$.

A slightly modified argument shows that $I^{+}(v)=\left\langle v^{*} u^{+}, \mu_{M}\right\rangle$.

\section{The secondary Chern-Euler class}

For a precise definition of Sha's secondary Chern-Euler class, $\Upsilon \in H^{m-1}\left(T M_{0}^{\partial}\right.$; $\mathrm{Z}\left[\frac{1}{2}\right]$ ), we refer to [4]. Here we will only use one of the properties of $\Upsilon$ to relate it to the index classes.

THeorem 5.1 (Sha). Let $v$ be a vector field on a manifold $M$ which is nonvanishing on the boundary and has isolated zeros. Then

$$
\left\langle v^{*} \Upsilon, \mu_{\partial M}\right\rangle= \begin{cases}\chi(M)-I^{-}(v) & \text { if } m \text { is odd } \\ -I^{-}(v) & \text { if } m \text { is even. }\end{cases}
$$

Proof of Theorem 1.2. Using the Poincaré-Hopf theorem we observe that

$$
I^{\circ}(v)+I^{-}(v)=I^{\circ}(-v)+I^{-}(-v)=(-1)^{m} I^{\circ}(v)+(-1)^{m-1} I^{+}(v) .
$$

Now consider the class $\tilde{u}^{+}-\tilde{u}^{-}$, using (5.2) we have

$$
\begin{aligned}
\left\langle v^{*}\left(\tilde{u}^{+}-\tilde{u}^{-}\right), \mu_{\partial M}\right\rangle & =\left\langle v^{*}\left(\tilde{u}^{+}-\tilde{u}^{-}\right), \partial \mu_{M}\right\rangle \\
& =\left\langle v^{*} \delta\left(\tilde{u}^{+}-\tilde{u}^{-}\right), \mu_{M}\right\rangle \\
& =\left\langle v^{*}\left(u^{+}-u^{-}\right), \mu_{M}\right\rangle \\
& =I^{+}(v)-I^{-}(-v) \\
& =2 \begin{cases}\chi(M)-I^{-}(v) & \text { if } m \text { is odd } \\
-I^{-}(v) & \text { if } m \text { is even. }\end{cases}
\end{aligned}
$$

The following lemma now finishes the proof. 
LemMA 5.2. Let $a \in H^{m-1}\left(T M_{0}^{\partial}\right)$. If $\left\langle v^{*} a, \mu_{\partial M}\right\rangle=0$ for all vector fields $v$ which are nonvanishing on the boundary, then $a=0$.

Proof. Without loss of generality we can assume $\partial M$ has one component. From the depicted maps we see that the short exact sequence for $\left(T M^{\partial}, T M_{0}^{\partial}\right)$ in the following diagram splits.

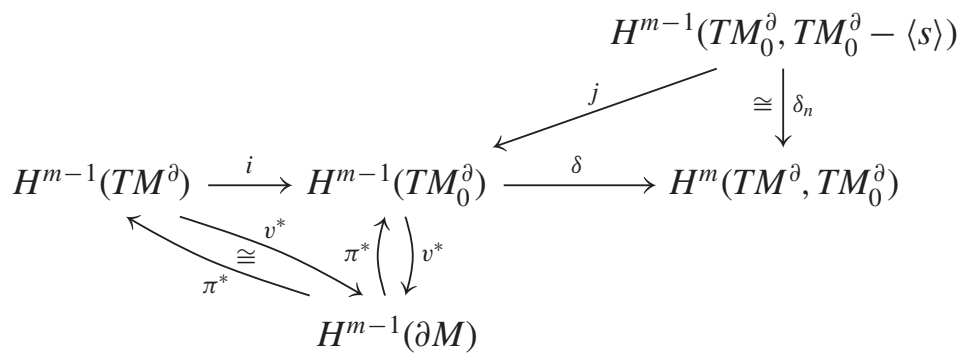

Thus every class $a \in H^{m-1}\left(T M_{0}^{\partial}\right)$ can be written uniquely as $a=a_{1}+a_{2}$ where $a_{1} \in \operatorname{Im}(i)$ and $a_{2} \in \operatorname{Im}(j)$. The group $H^{m-1}\left(T M_{0}^{\partial}, T M_{0}^{\partial}-\langle s\rangle\right)$ is generated by $\tilde{u}$, the preimage of $\tilde{u}^{-}$. Choose a generator $\zeta$ for $H^{m-1}\left(T M^{\partial}\right)$. We can then write $a$ as $a=a_{1}+a_{2}=i p \zeta+j q \tilde{u}$, where $p, q \in \mathrm{Z}$. The lower triangle commutes so $v^{*} a_{1}=w^{*} a_{1}$ for all vector fields $v, w$. We know that $\left\langle v^{*} j \tilde{u}, \mu_{\partial M}\right\rangle=\operatorname{Ind}^{-}(v)$. We can construct two vector fields $v_{0}$ and $v_{1}$ such that $\left\langle v_{k}^{*} j \tilde{u}, \mu_{\partial M}\right\rangle=\operatorname{Ind}^{-}\left(v_{k}\right)=k$ for $k=0,1$. Applying these vector fields to a class $a_{1}+a_{2} \in \operatorname{Ker}\left(v^{*}\right)$ for all $v$ we see that

$$
0=\left\langle v_{k}^{*}\left(a_{1}+a_{2}\right), \mu_{\partial M}\right\rangle=\left\langle v_{k}^{*}(i p \zeta+j q \tilde{u}), \mu_{\partial M}\right\rangle=p+k q,
$$

for $k=0$, which implies that $p=0$ and $q=0$, thus $a=a_{1}+a_{2}=$ $i p \chi+j q \tilde{u}=0$.

\section{REFERENCES}

1. Gottlieb, D. H., A de Moivre like formula for fixed point theory, pp. 99-105 in: Fixed Point Theory and its Applications, Proc. Berkeley 1986, Contemp. Math. 72, Amer. Math. Soc., Providence, RI 1988.

2. Morse, Marston, Singular points of vector fields under general boundary conditions, Amer. J. Math. 51 (1929), 166-178.

3. Pugh, C. C., A generalized Poincaré index formula, Topology 7 (1968), 217-226.

4. Sha, Ji-Ping, A secondary Chern-Euler class, Ann. of Math. (2) 45 (1999), 1151-1158. 
5. Sharafutdinov, V. A., Relative Euler class and the Gauss-Bonnet theorem, Sibirsk. Mat. Zh. 14 (1973), 1321-1335.

MATEMATISKA VETENSKAPER

CHALMERS TEKNISKA HÖGSKOLA

OCH GÖTEBORGS UNIVERSITET

SE-412 96 GÖTEBORG

SWEDEN

E-mail: hamlet@chalmers.se 